\title{
The association between masers and outflows in massive star forming regions
}

\author{
C. Codella ${ }^{1}$, A. Lorenzani ${ }^{1}$, A. T. Gallego ${ }^{2}$, R. Cesaroni ${ }^{3}$, and L. Moscadelli ${ }^{4}$ \\ ${ }^{1}$ Istituto di Radioastronomia, CNR, Sezione di Firenze, Largo E. Fermi 5, 50125 Firenze, Italy \\ 2 IRAM, Avda Divina Pastora 7, Núcleo Central, 18012 Granada, Spain \\ 3 INAF, Osservatorio Astrofisico di Arcetri, Largo E. Fermi 5, 50125 Firenze, Italy \\ ${ }^{4}$ INAF, Osservatorio Astronomico di Cagliari, Loc. Poggio dei Pini, 09012 Capoterra (Cagliari), Italy
}

Received 31 October 2003 / Accepted 5 December 2003

\begin{abstract}
We report the results of a single-dish survey of molecular outflows towards a homogeneous sample of 136 ultracompact $\mathrm{H}$ II regions for which we had previously obtained observations in the methanol $6.7 \mathrm{GHz}$ and water $22.2 \mathrm{GHz}$ maser lines. The line profiles of the ${ }^{13} \mathrm{CO} J=1-0$ and $2-1$ transitions have been compared to those of the corresponding lines of the $\mathrm{C}^{18} \mathrm{O}$ isotopomer to reveal the occurrence of line wings and hence of molecular outflows. We found 53 outflows resulting in an overall detection rate of $\sim 39 \%$. The probability to have an outflow increases to about $50 \%$ in regions with maser emission, whereas it is about $25 \%$ in those without masers. If we consider just the outflow sources, the chance to find a maser is very high: 74\%, without a significant difference between $\mathrm{H}_{2} \mathrm{O}$ and $\mathrm{CH}_{3} \mathrm{OH}$. These results strongly confirm from a statistical point of view that both types of masers are closely associated with the evolutionary phase when outflows occur.

The temperatures and optical depths of the molecular cloud hosting the ultracompact $\mathrm{H}$ II regions and the comparison between the detection rates suggest a tentative evolutionary scheme for massive star forming regions: the earliest phase is associated with maser emission and with an outflow not yet developed enough to be detected with single-dish observations; then maser emission disappears while the outflow is still present; and finally, only the ultracompact HII region without masers or outflows is present.
\end{abstract}

Key words. stars: formation - radio lines: ISM - ISM: jets and outflows - ISM: molecules

\section{Introduction}

It is well known that water $\left(\mathrm{H}_{2} \mathrm{O}\right)$ and Class II methanol $\left(\mathrm{CH}_{3} \mathrm{OH}\right)$ maser transitions are commonly observed towards regions of high-mass star formation, the strongest emission lines being those at $22.2 \mathrm{GHz}$ for water and at $6.7 \mathrm{GHz}$ for methanol. Like other interstellar masers, $\mathrm{H}_{2} \mathrm{O}$ and $\mathrm{CH}_{3} \mathrm{OH}$ can be used as signposts of optically obscured regions where the earliest phases of the star forming process occurs.

The evolutionary stage associated with $22.2 \mathrm{GHz}$ $\mathrm{H}_{2} \mathrm{O}$ maser emission has been investigated through extensive single-dish and high-angular resolution observations (e.g. Tofani et al. 1995; Codella et al. 1997; Kurtz et al. 2000), which have indicated that this type of maser may be associated with very young massive (proto-)stars prior to the development of an ultracompact (UC) HII region. Although in a few cases it has been claimed that $\mathrm{H}_{2} \mathrm{O}$ masers could trace circumstellar disks around (proto-)stellar objects (Cesaroni 1990; Torrelles et al. 1997; Seth et al. 2002), interferometric and VLBI observations towards high-mass forming stars have shown that

Send offprint requests to: C. Codella, e-mail: codella@arcetri.astro.it water masers are predominantly associated with jets and outflows (e.g. Moscadelli et al. 2000; Torrelles et al. 2001).

On the other hand, to date it is not clear when the $6.7 \mathrm{GHz}$ phase occurs. Although blind searches have been performed towards the Galactic plane (e.g. Szymczak et al. 2000), most of the observations have been focused on samples of IRAS point sources and UCHII regions (Caswell et al. 1995; Ellingsen et al. 1996; Slysh et al. 1999). Thanks to high angular resolution observations, Walsh et al. $(1998,2003)$ and Beuther et al. (2002b) compared the positions of $\mathrm{CH}_{3} \mathrm{OH}$ masers with other tracers of star formation such as (sub-)mm and cm continuum emission, mid-infrared sources, and $\mathrm{H}_{2} \mathrm{O}$ masers. Their results suggest that also the $6.7 \mathrm{GHz}$ emission might trace a pre-UCH II region phase and disappear as the UCH II region evolves. This picture is statistically supported also by the survey performed by Codella \& Moscadelli (2000), who have demonstrated that the 6.7 and $22.2 \mathrm{GHz}$ masers are associated with two at least partly overlapping evolutionary phases. Interferometric and VLBI observations have shown that the $6.7 \mathrm{GHz}$ maser spots are often distributed along lines or arcs of size from 100 mas to 1 arcsec with, occasionally, a monotonic velocity trend: this suggests that the methanol masers trace 
Table 1. List of transitions and observational parameters: Angular resolution, system temperature, and spectral resolutions.

\begin{tabular}{ccccccc}
\hline \hline Molecule & Transition & $\begin{array}{c}\text { Rest frequency } \\
(\mathrm{MHz})\end{array}$ & $\begin{array}{c}H P B W \\
\left({ }^{\prime \prime}\right)\end{array}$ & $\begin{array}{c}T_{\text {sys }} \\
(\mathrm{K})\end{array}$ & $\begin{array}{c}d v(\mathrm{AC}) \\
\left(\mathrm{km} \mathrm{s}^{-1}\right)\end{array}$ & $\begin{array}{c}d v(\mathrm{FB}) \\
\left(\mathrm{km} \mathrm{s}^{-1}\right)\end{array}$ \\
\hline $\mathrm{C}^{18} \mathrm{O}$ & $J=1-0$ & 109782.156 & 22 & 150 & 0.21 & 2.73 \\
${ }^{13} \mathrm{CO}$ & $J=1-0$ & 110201.352 & 22 & 200 & 0.21 & 2.72 \\
$\mathrm{C}^{18} \mathrm{O}$ & $J=2-1$ & 219560.328 & 11 & 300 & 0.11 & 1.37 \\
${ }^{13} \mathrm{CO}$ & $J=2-1$ & 220398.688 & 11 & 300 & 0.11 & 1.36 \\
\hline
\end{tabular}

ordered motions (Norris et al. 1998; Minier et al. 2000), which could originate from circumstellar accretion disks.

So far, most high-resolution studies have focused either on $\mathrm{CH}_{3} \mathrm{OH}$ or on $\mathrm{H}_{2} \mathrm{O}$ masers and little is known about their connection, whereas it would be important to study both types of maser emission in the same object. Single-dish observations show that, for sources in which both maser species coexist, the velocity spread is by far larger for $\mathrm{H}_{2} \mathrm{O}$ (up to $100 \mathrm{~km} \mathrm{~s}^{-1}$ ) than for $\mathrm{CH}_{3} \mathrm{OH}$ masers $\left(<20 \mathrm{~km} \mathrm{~s}^{-1}\right)($ Codella \& Moscadelli 2000; Sridharan et al. 2002): this suggests that the two maser types could originate from different environments. This topic was further investigated by Beuther et al. (2002b) who found that, probably due to different excitation processes, no spatial correlation between $\mathrm{CH}_{3} \mathrm{OH}$ and $\mathrm{H}_{2} \mathrm{O}$ exist. For example, a possible interpretation might be that the $\mathrm{CH}_{3} \mathrm{OH}$ masers are confined to the innermost part of the environment of the forming star (tracing the root of a jet or possibly a disk), whereas the $\mathrm{H}_{2} \mathrm{O}$ maser emission originates in a larger scale region, associated with the high-velocity part of the accelerated jet.

In summary, (i) both $\mathrm{CH}_{3} \mathrm{OH}$ and $\mathrm{H}_{2} \mathrm{O}$ masers are associated with the earliest phases of the formation of massive stars; (ii) both types of masers seem to disappear during the development of an UCHII region; (iii) $\mathrm{H}_{2} \mathrm{O}$ masers seem to be directly associated with molecular outflows, whereas a similar result is still under debate for $\mathrm{CH}_{3} \mathrm{OH}$ masers. Molecular outflows might represent the key to locate the two types of masers in the evolutionary sequence leading to high-mass star formation, since they are associated with the earliest evolutionary phases and tend to disappear once the star has reached the zero-age main sequence. With this in mind, one may reasonably use the outflow detection rate as an estimate of the age of the associated sources. In the present paper we present observations of the $J=1-0$ and 2-1 transitions of ${ }^{13} \mathrm{CO}$, which can trace the outflows, and $\mathrm{C}^{18} \mathrm{O}$, which traces the ambient gas. In particular, we investigate high-mass star forming regions in a relatively advanced stage of their evolution as witnessed by their association with UCHII regions: consequently we focus our attention on the evolutionary phases when masers and outflows are progressively fading away. Our goal is to clarify when masers switch off and to check whether the outflow detection rate varies depending on the presence of the $6.7 \mathrm{GHz}$ and/or the $22.2 \mathrm{GHz}$ masers.

\section{The sample}

To reach the aim described in the previous section, we need a homogeneous sample of young stellar objects (YSOs) for which the existence of $\mathrm{CH}_{3} \mathrm{OH}$ and $\mathrm{H}_{2} \mathrm{O}$ maser emission has been assessed. This has been provided by Codella \& Moscadelli (2000), who performed observations at 6.7 and $22.2 \mathrm{GHz}$ towards a sample of massive star forming regions with the 32-m Medicina (Italy) antenna. They used the unbiased $1.4 \mathrm{GHz}$ continuum survey of Zoonematkermani et al. (1990), who observed a $140^{\circ} \times 1.6^{\circ}$ strip of the Galactic plane with the VLA, detecting discrete sources down to a limit flux of about $10 \mathrm{mJy}$. The cross-correlation between the objects detected at $1.4 \mathrm{GHz}$ and the IRAS Point Source Catalogue has allowed the selection of a sample of $166 \mathrm{UCH}$ II regions (White et al. 1991), which offers a precious opportunity to perform statistical investigations. The observations by Codella \& Moscadelli (2000) resulted in almost identical detection rates for the $\mathrm{CH}_{3} \mathrm{OH}$ and $\mathrm{H}_{2} \mathrm{O}$ masers: $23 \%$ and $22 \%$, respectively. For the present ${ }^{13} \mathrm{CO}$ and $\mathrm{C}^{18} \mathrm{O}$ observations, in practice our list was limited to $136 \mathrm{UCH}$ II regions due to constraints such as the declination of the sources and time limitations.

\section{Observations}

The observations were carried out with the IRAM 30-m telescope at Pico Veleta (Granada, Spain) during several runs in April and August 2000. Table 1 summarises the observed molecular species, the transitions, and their rest frequencies. The main beam efficiency varies from 0.80 (at $110 \mathrm{GHz}$ ) to 0.52 (at $220 \mathrm{GHz}$ ). The observations were made in position switching. Pointing was checked about every hour by observing nearby planets or continuum sources and was found to be accurate within $4^{\prime \prime}$. An autocorrelator (AC) spectrometer split into four parts was used to allow simultaneous observations of four different transitions. Moreover, a $1 \mathrm{MHz}$ filter bank (FB), split into four parts of 256 channels, was simultaneously used. The integration time $\mathrm{ON}+\mathrm{OFF}$ source is $120 \mathrm{~s}$. The velocity resolutions provided by both backends, $\mathrm{AC}$ and $\mathrm{FB}$, as well as the $H P B W$ and the typical system temperature $\left(T_{\text {sys }}\right)$, are also shown in Table 1 . The average rms in the spectra is about 80 and $500 \mathrm{mK}$ at 110 and $220 \mathrm{GHz}$, respectively. The spectra were calibrated with the standard chopper wheel method and are reported here in units of main-beam brightness temperature $\left(T_{\mathrm{MB}}\right)$.

\section{Results and discussion}

The results reported below could be affected by the different angular resolutions used in the maser and $\mathrm{CO}$ observations. In fact, while the separation between two adjacent objects is in 


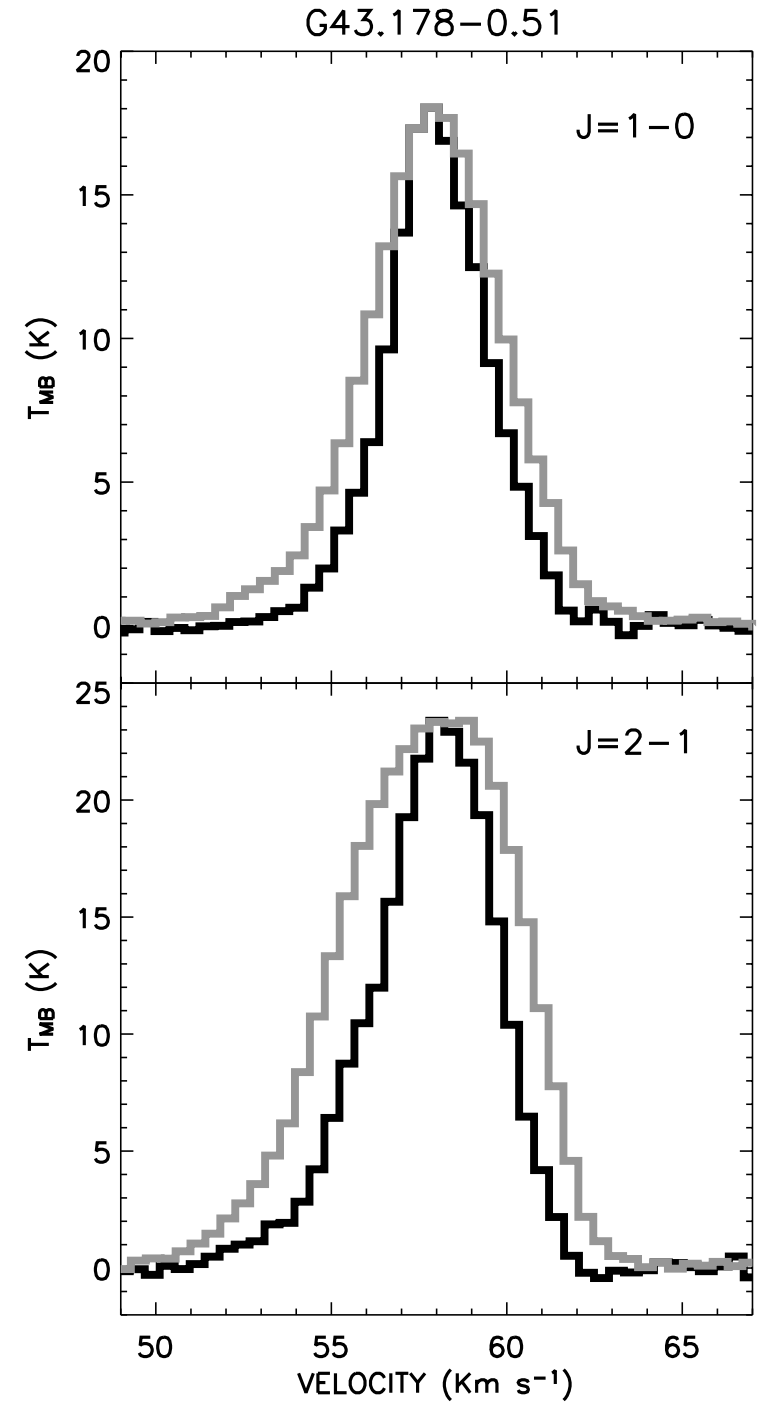

Fig. 1. Examples of the observed ${ }^{13} \mathrm{CO}$ (gray contours) and $\mathrm{C}^{18} \mathrm{O}$ (black contours) $J=1-0$ (upper panel) and 2-1 (lower panel) spectra. The $\mathrm{C}^{18} \mathrm{O}$ have been rescaled to be comparable with the ${ }^{13} \mathrm{CO}$ ones. The average scaling factors are 4.7 and 4.3 for the $J=1-0$ and 2-1 transitions, respectively. A typical case where the detection of red and blue ${ }^{13} \mathrm{CO}$ wings indicate the occurrence of outflows motions is reported.

all cases larger than the $H P B W \mathrm{~s}$ of the 30-m IRAM telescope, 38 sources (28\%) lie closer to another object of our list than the $H P B W \mathrm{~s}$ of the 32-m Medicina antenna. Thus, the statistics about detection rates, distances, and gas parameters has been re-analysed using only those UCHII regions which do not have other objects of the sample within the Medicina HPBWs. The results calculated with the remaining 98 objects indicates that no bias has been introduced by the use of large $H P B W$ s at centimeter wavelengths.

\subsection{Detection rates}

Figure 1 reports examples of the observed spectra. $\mathrm{C}^{18} \mathrm{O}$ is a good tracer of the quiescent ambient molecular gas whereas ${ }^{13} \mathrm{CO}$ is one of the tools to detect molecular outflows,
Table 2. Results of the outflow survey towards UCH II regions.

\begin{tabular}{ccc}
\hline \hline Sources & Number & With outflow \\
\hline Masers & 80 & $39(49 \%)$ \\
no Masers & 56 & $14(25 \%)$ \\
\hline $\mathrm{H}_{2} \mathrm{O}$ & 63 & $31(49 \%)$ \\
no $\mathrm{H}_{2} \mathrm{O}$ & 73 & $22(30 \%)$ \\
\hline $\mathrm{CH}_{3} \mathrm{OH}$ & 47 & $24(51 \%)$ \\
no $\mathrm{CH}_{3} \mathrm{OH}$ & 89 & $29(33 \%)$ \\
\hline Total & 136 & $53(39 \%)$ \\
\hline
\end{tabular}

especially in crowded high-mass star forming regions where ${ }^{12} \mathrm{CO}$ can be seriously affected by confusion. We compared the line profiles of the same transition for the two species to detect the occurrence of line wings and hence of molecular outflows. In practice, the $\mathrm{C}^{18} \mathrm{O}$ profile was scaled to the same peak intensity as the ${ }^{13} \mathrm{CO}$ one and then substracted from it. We assume occurrence of an outflow if at least one of the observed ${ }^{13} \mathrm{CO}$ transitions shows residuals in the wings at a level $\geq 3 \sigma$. As an example, Fig. 1 presents a comparison between the profiles of the $\mathrm{C}^{18} \mathrm{O}$ and ${ }^{13} \mathrm{CO}$ lines in an UCH II region (G43.178-0.518) where broad wings have been detected. This method to search for outflows is affected by errors due to confusion (the observed sources lie along the Galactic plane), spectral noise (in case of weak sources) and outflow geometry (which determines the width of the wings in the profile). The consequence is that we may miss a few detections, but given the homogeneity of the present sample and the large number of the observed objects, there is no reason why this bias should be more relevant in one of the subsamples (e.g. water masers) than in others. In particular, the reliability of the present study has been checked by cross-correlating our results with those of the ${ }^{12} \mathrm{CO}$ outflow survey by Shepherd \& Churchwell (1996a). The regions in common are 24 (18\% of our sample) and the check indicates that: (i) in all cases our ${ }^{13} \mathrm{CO}$ outflow detections are confirmed by the ${ }^{12} \mathrm{CO}$ data, and (ii) the ${ }^{12} \mathrm{CO}$ line widths of the objects without ${ }^{13} \mathrm{CO}$ outflows are on average smaller than those measured in regions associated with a ${ }^{13} \mathrm{CO}$ outflow. These results confirm the validity of our method.

Table 2 summarises the results of the survey for the whole sample, while Table 3 reports names and coordinates and indicates if the observed sources are associated or not with $\mathrm{CH}_{3} \mathrm{OH}$ or $\mathrm{H}_{2} \mathrm{O}$ masers (Codella \& Moscadelli 2000), and outflows. Out of the observed $136 \mathrm{UCH}$ II regions, 53 show the occurrence of a CO outflow. The overall detection rate is thus $39 \%$. This value is lower with respect to those found in ${ }^{12} \mathrm{CO}$ outflow surveys towards massive star forming regions: e.g. Zhang et al. (2001) report a detection rate of $60 \%$ for a sample of luminous IRAS sources, while Sridharan et al. (2002) and Shepherd \& Churchwell (1996a) have a detection rate of $\sim 90 \%$ for their sample of UCHII regions. However, this apparent discrepancy can be attributed to two reasons. First of all, as discussed above, line wings are less prominent in ${ }^{13} \mathrm{CO}$ than in ${ }^{12} \mathrm{CO}$, hence the outflow detection rate is expected to be lower. Moreover, as noted by Zhang et al. (2001), the detection rate, probably 
Table 3. List of the sources surveyed. "Met", "Wat", and "Out" refer to the detection of $\mathrm{CH}_{3} \mathrm{OH}, \mathrm{H}_{2} \mathrm{O}$ masers and outflows, respectively.

\begin{tabular}{|c|c|c|c|c|c|c|c|}
\hline Source & $\begin{array}{c}\alpha_{1950} \\
(\mathrm{~h} \mathrm{~m} \mathrm{~s})\end{array}$ & $\begin{array}{l}\delta_{1950} \\
\left({ }^{\circ}, \prime \prime\right)\end{array}$ & Met? Wat? Out? & Source & $\begin{array}{c}\alpha_{1950} \\
(\mathrm{~h} \mathrm{~m} \mathrm{~s})\end{array}$ & $\begin{array}{c}\delta_{1950} \\
\left({ }^{\circ}, \prime \prime\right)\end{array}$ & Met? Wat? Out? \\
\hline G0.279-0.482 & 174459.4 & -285551 & N N N & G16.945-0.073 & 181905.4 & -141455 & $\mathrm{NNN}$ \\
\hline G0.668-0.035 & 174410.6 & -282159 & Y Y Y & G18.147-0.283 & 182211.5 & -131717 & $\mathrm{~N} \mathrm{NN}$ \\
\hline G0.669-0.028 & 174409.1 & -282142 & Y Y Y & G18.304-0.390 & 182253.0 & -131203 & N N Y \\
\hline G0.671-0.036 & 174411.1 & -282152 & Y Y Y & G18.461-0.004 & 182147.4 & -125246 & Y N N \\
\hline G1.127-0.106 & 174532.2 & -280042 & Y Y Y & G18.833-0.300 & 182334.9 & -124127 & Y N N \\
\hline $\mathrm{G} 2.615+0.134$ & 174804.9 & -263653 & Y N N & G19.076-0.287 & 182400.0 & -122815 & N Y Y \\
\hline $\mathrm{G} 2.894+0.025$ & 174908.7 & -262554 & N N N & G19.078-0.288 & 182400.5 & -122811 & N Y Y \\
\hline G3.349-0.075 & 175034.2 & -260531 & $\mathrm{~N} \mathrm{NN}$ & G19.491+0.135 & 182316.6 & -115420 & Y N N \\
\hline G3.352-0.077 & 175034.9 & -260528 & N N N & G19.611-0.234 & 182450.3 & -115827 & Y Y N \\
\hline G3.439-0.348 & 175149.1 & -260918 & N N Y & G20.072-0.142 & 182523.5 & -113123 & Y Y N \\
\hline $\mathrm{G} 3.721+0.016$ & 175103.9 & -254334 & N N N & G20.081-0.136 & 182523.1 & -113045 & Y Y N \\
\hline G3.928-0.116 & 175202.4 & -253655 & $\mathrm{~N} \mathrm{NN}$ & G20.989+0.090 & 182618.5 & -103615 & $\mathrm{~N} \mathrm{NN}$ \\
\hline $\mathrm{G} 4.417+0.125$ & 175213.3 & -250417 & N N N & G21.874+0.008 & 182817.2 & -095138 & Y Y Y \\
\hline G5.476-0.243 & 175558.7 & -242040 & $\mathrm{~N} \mathrm{NN}$ & G23.265+0.077 & 183040.2 & -083552 & N Y N \\
\hline G5.639+0.237 & 175431.3 & -235743 & N N Y & G23.439-0.210 & 183201.3 & -083440 & Y N N \\
\hline G5.884-0.398 & 175727.9 & -240412 & Y Y N & $\mathrm{G} 23.711+0.171$ & 183110.1 & -080934 & N Y Y \\
\hline G5.885-0.392 & 175726.8 & -240356 & Y Y Y & G23.873-0.119 & 183230.8 & -080905 & $\mathrm{~N} \mathrm{NN}$ \\
\hline G6.552-0.098 & 175747.4 & -232029 & Y Y N & $\mathrm{G} 23.956+0.150$ & 183142.3 & -075710 & N Y Y \\
\hline G6.553-0.095 & 175747.0 & -232020 & Y Y N & $\mathrm{G} 24.467+0.490$ & 183127.0 & -072030 & N Y Y \\
\hline $\mathrm{G} 7.471+0.063$ & 175910.7 & -222753 & N Y N & G24.507-0.222 & 183404.1 & -073813 & N N N \\
\hline G7.474+0.064 & 175911.0 & -222741 & N Y N & G25.306+0.531 & 183252.5 & -063449 & N N Y \\
\hline G8.140-0.027 & 180057.2 & -215545 & N Y N & $\mathrm{G} 25.398+0.562$ & 183256.2 & -062904 & N N Y \\
\hline G8.141+0.224 & 180000.9 & -214813 & N N N & G25.460-0.208 & 183548.1 & -064711 & N N Y \\
\hline G9.617+0.196 & 180315.3 & -203205 & Y Y Y & G26.545+0.415 & 183536.0 & -053210 & N N Y \\
\hline G9.623+0.198 & 180315.5 & -203144 & Y Y Y & G26.597-0.023 & 183715.6 & -054134 & Y N Y \\
\hline G10.320-0.156 & 180602.1 & -200541 & Y Y Y & $\mathrm{G} 27.978+0.078$ & 183927.6 & -042517 & $\mathrm{~N} \mathrm{NN}$ \\
\hline G10.462+0.034 & 180537.6 & -195241 & Y Y N & G28.199-0.050 & 184019.5 & -041705 & Y N Y \\
\hline G10.959+0.022 & 180642.0 & -192703 & Y N N & G28.201-0.049 & 184019.4 & -041657 & Y N Y \\
\hline G10.965+0.009 & 180645.8 & -192705 & Y N Y & $\mathrm{G} 28.245+0.014$ & 184010.9 & -041250 & $\mathrm{~N} \mathrm{NN}$ \\
\hline G11.034+0.063 & 180642.4 & -192155 & Y Y N & G28.288-0.364 & 184136.3 & -042101 & Y N Y \\
\hline G11.110-0.399 & 180834.5 & -193124 & $\mathrm{~N} \mathrm{NN}$ & $\mathrm{G} 28.609+0.018$ & 184050.3 & -035322 & $\mathrm{~N} \mathrm{NN}$ \\
\hline G11.941-0.042 & 180857.6 & -183726 & $\mathrm{~N} \mathrm{~N} \mathrm{~N}$ & $\mathrm{G} 28.802+0.172$ & 184038.9 & -033848 & $\mathrm{~N} \mathrm{NN}$ \\
\hline G11.945-0.037 & 180857.0 & -183705 & $\mathrm{~N} \mathrm{NN}$ & G30.778-0.015 & 184457.8 & -015848 & Y Y N \\
\hline G13.211-0.143 & 181155.0 & -173341 & N N N & G30.866+0.114 & 184439.8 & -015030 & Y Y N \\
\hline G13.385+0.067 & 181130.0 & -171828 & N N N & $\mathrm{G} 31.070+0.050$ & 184516.1 & -014125 & Y N N \\
\hline G13.874+0.281 & 181142.0 & -164631 & N Y Y & G31.243-0.109 & 184609.1 & -013637 & N Y N \\
\hline G15.075-0.121 & 181534.5 & -155454 & $\mathrm{~N} \mathrm{NN}$ & G31.280+0.063 & 184536.4 & -012954 & Y Y Y \\
\hline G16.311-0.476 & 181918.8 & -145950 & N N N & $\mathrm{G} 32.150+0.133$ & 184657.8 & -004135 & N Y N \\
\hline
\end{tabular}

due to confusion, depends on the galactic longitude ranging from to $90 \%$ for sources between $50^{\circ}<l<240^{\circ}$ to $15 \%$ in the region with $0^{\circ}<l<50^{\circ}$. Also for the sample used by Shepherd \& Churchwell (1996a) the occurrence of outflows decreases to about $50 \%$ because of the presence of complex spectra with many overlapping lines for sources with small longitudes. The present sample mostly contains UCHII regions with $l \leq 50^{\circ}(88 \%)$, and thus an overall detection rate of $39 \%$ can be considered in reasonable agreement with the previous surveys.

The probability to have an outflow increases to $\sim 50 \%$ in regions where maser emission $\left(6.7 \mathrm{GHz} \mathrm{CH} \mathrm{CH}_{3} \mathrm{OH}\right.$ and/or $22.2 \mathrm{GHz} \mathrm{H}_{2} \mathrm{O}$ ) has been observed, whereas it drops to about $25 \%$ in those without masers. On the other hand, if we 
Table 3. continued.

\begin{tabular}{|c|c|c|c|c|c|c|c|}
\hline Source & $\begin{array}{c}\alpha_{1950} \\
(\mathrm{~h} \mathrm{~m} \mathrm{~s})\end{array}$ & $\begin{array}{c}\delta_{1950} \\
\left({ }^{\circ}, \prime \prime\right)\end{array}$ & Met? Wat? Out? & Source & $\begin{array}{c}\alpha_{1950} \\
\left({ }^{\mathrm{h} \mathrm{m} \mathrm{s}}\right)\end{array}$ & $\begin{array}{c}\delta_{1950} \\
\left({ }^{\circ}, \prime \prime\right)\end{array}$ & Met? Wat? Out? \\
\hline G32.273-0.225 & 184827.7 & -004457 & N N Y & G43.890-0.783 & 191202.8 & +091722 & Y Y Y \\
\hline G32.473+0.204 & 184718.3 & -002227 & N N Y & G45.123+0.132 & 191106.4 & +104826 & Y N Y \\
\hline G32.799+0.191 & 184757.2 & -000527 & N Y N & G45.129+0.131 & 191107.3 & +104843 & Y N N \\
\hline G32.928+0.607 & 184642.8 & +001256 & N N N & G45.454+0.060 & 191159.9 & +110358 & Y Y Y \\
\hline G32.991+0039 & 184850.7 & +000038 & Y N Y & G45.466+0.046 & 191204.3 & +110412 & Y Y Y \\
\hline G33.133-0.092 & 184934.4 & +000433 & Y Y Y & G45.477+0.130 & 191147.4 & +110711 & Y Y Y \\
\hline G33.142-0.086 & 184934.0 & +000512 & Y Y Y & G45.480+0.136 & 191146.5 & +110729 & Y Y N \\
\hline G34.089+0.436 & 184927.3 & +011004 & N N N & G45.543-0.007 & 191224.4 & +110649 & N N Y \\
\hline G34.133+0.471 & 184924.8 & +011322 & N N Y & G48.592+0.044 & 191806.8 & +134935 & N Y N \\
\hline G34.198-0.591 & 185318.3 & +004730 & N N N & G48.603+0.026 & 191811.9 & +134942 & N Y N \\
\hline G34.255+0.146 & 185047.5 & +011055 & Y Y N & G48.606+0.023 & 191812.9 & +134944 & N Y N \\
\hline G35.052-0.518 & 185437.1 & +013501 & N Y N & G48.609+0.027 & 191812.5 & +135002 & N Y N \\
\hline G35.467+0.139 & 185303.0 & +021515 & N N N & G50.286-0.393 & 192301.8 & +150629 & N N N \\
\hline G35.573+0.068 & 185329.8 & +021856 & N N Y & G50.316+0.676 & 191911.5 & +153839 & N Y Y \\
\hline G35.578-0.030 & 185351.4 & +021630 & N Y N & G52.753+0.335 & 192518.3 & +173720 & N N N \\
\hline $\mathrm{G} 37.821+0.413$ & 185625.2 & +042810 & N Y Y & G53.959+0.032 & 192852.2 & +183153 & N N N \\
\hline G37.874-0.399 & 185924.4 & +040831 & N Y Y & G57.548-0.272 & 193729.8 & +213034 & $\mathrm{~N} \mathrm{~N} \mathrm{~N}$ \\
\hline G38.549+0.163 & 185839.5 & +050003 & N N N & G60.882-0.132 & 194414.3 & +242751 & N Y Y \\
\hline G38.694-0.452 & 190107.1 & +045038 & $\mathrm{~N} \mathrm{~N} \mathrm{~N}$ & G61.474+0.093 & 194442.6 & +250455 & N Y Y \\
\hline G38.876+0.308 & 185844.8 & +052125 & N N N & G74.792+0.580 & 201603.9 & +363617 & N N N \\
\hline G39.249-0.066 & 190046.5 & +053054 & N Y N & G75.768+0.342 & 201949.8 & +371615 & Y Y N \\
\hline G39.727-0.397 & 190250.6 & +054709 & N N Y & G76.188+0.098 & 202203.0 & +372825 & N N N \\
\hline G39.883-0.346 & 190257.1 & +055651 & N N Y & G76.384-0.622 & 202534.1 & +371253 & N Y N \\
\hline G40.424+0.699 & 190013.8 & +065440 & Y N N & G77.965-0.007 & 202746.2 & +385114 & N N N \\
\hline G41.513-0.141 & 190516.0 & +072912 & N N N & G79.307+0.277 & 203043.2 & +400608 & N N N \\
\hline G41.742+0.097 & 190450.6 & +074800 & N N N & G80.865+0.420 & 203504.4 & +412554 & Y N Y \\
\hline G43.170+0.007 & 190751.1 & +090122 & Y Y N & G105.627+0.339 & 223052.6 & +581251 & N Y Y \\
\hline G43.178-0.518 & 190944.9 & +084702 & N Y Y & G108.197+0.579 & 224730.8 & +593859 & N Y Y \\
\hline G43.186-0.526 & 190947.5 & +084716 & N Y N & G110.109+0.047 & 230304.9 & +595835 & N Y N \\
\hline G43.237-0.045 & 190809.8 & +090328 & N Y Y & G111.284-0.664 & 231353.5 & +594539 & N Y N \\
\hline
\end{tabular}

consider the outflow sources, the chance to find a maser is very high: $74 \%$. The outflow detection rate is quite high in the two subsamples composed by the UCH II regions associated with either water or methanol masers: $\sim 50 \%$ in both cases. These two values are equal, within the statistical uncertainty, which indicates that the two types of masers trace roughly the same evolutionary stage. In the following, we will use the word maser to refer to sources with any type of maser, unless otherwise explicitly stated. These results confirm from a statistical point of view that masers are closely associated with outflows and thus with the earliest phases of the high-mass star forming process. However, taking into account that maps of the observed regions are not available, one cannot exclude that masers are produced in accretion disks around the YSOs powering the outflows.

\subsection{Distances and luminosities}

The kinematic distances have been obtained from the $\mathrm{C}^{18} \mathrm{O}$ line using the rotation curve of Brand (1986), which has an orbital velocity of $220 \mathrm{~km} \mathrm{~s}^{-1}$ at the Sun orbital radius $(8.5 \mathrm{kpc})$. To resolve the near/far distance ambiguity within the solar circle, distances have been compared with those given in the catalogues of Solomon et al. (1987), Downes et al. (1980), Wink et al. (1982), Araya et al. (2002), and Watson et al. (2003). Distance estimates for 58 UCHII regions have been obtained. For these regions, following the procedure described by Henning et al. (1990, see their Eqs. (4) and (5)) the integrated IRAS fluxes and consequently the IRAS luminosities $\left(L_{\mathrm{FIR}}\right)$ have been calculated by integrating the energy distribution between 7 and $140 \mu \mathrm{m}$ as the limiting wavelengths 

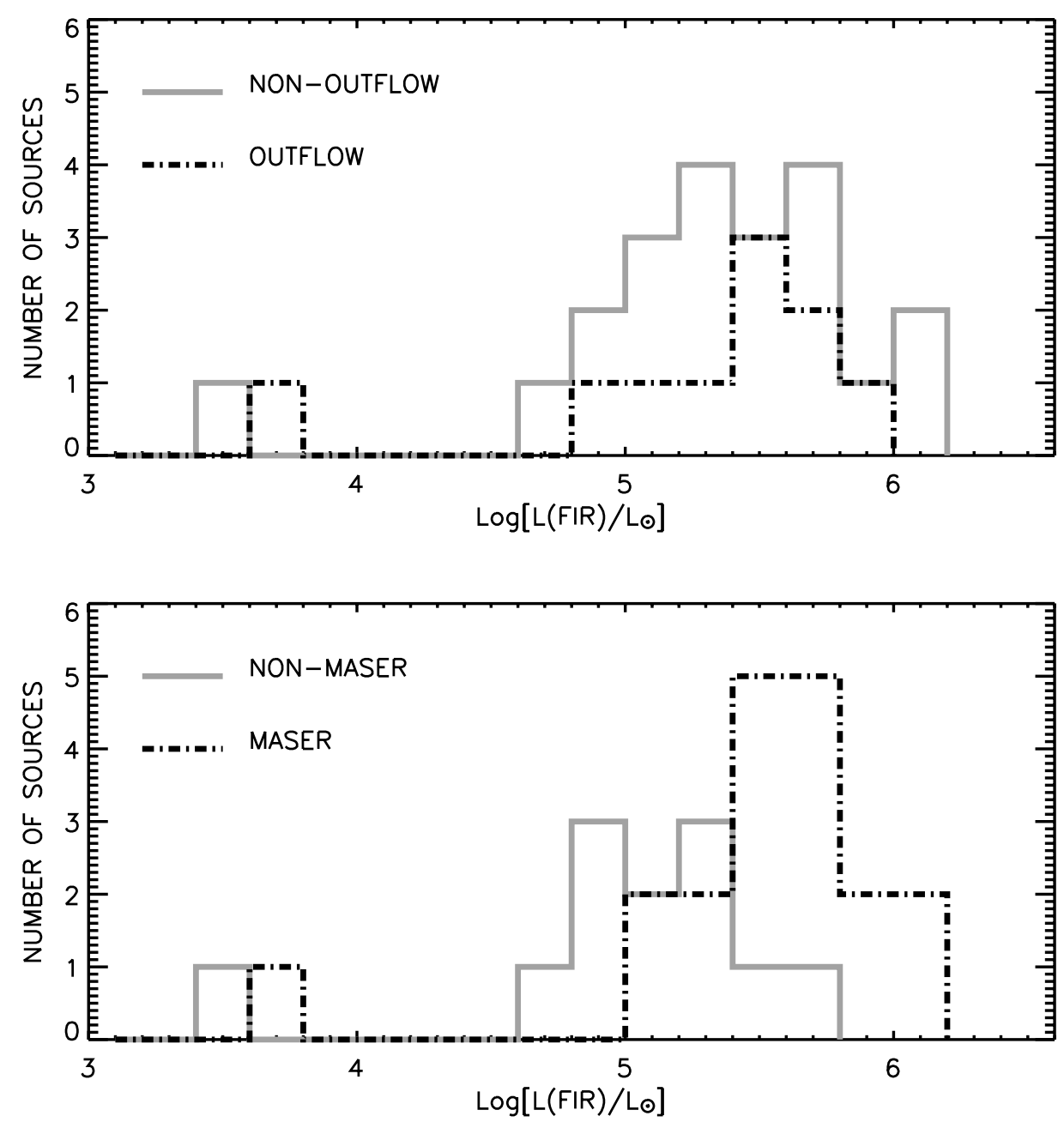

Fig. 2. Distributions of the FIR luminosity. The upper panel reports the distributions of the outflow (dot-dashed contour) and non-outflow (continuous) subsamples. The lower panel shows the distributions of the maser $\left(\mathrm{CH}_{3} \mathrm{OH}\right.$ and/or $\mathrm{H}_{2} \mathrm{O}$; dot-dashed) and non-maser (continuous) subsamples.

of the IRAS band-passes. The luminosity distributions of four subsamples, outflow, non-outflow, and maser, non-maser have been obtained and reported in Fig. 2: it is possible to see that the sources of the four subsamples have approximately the same luminosities, ranging from $4 \times 10^{3}$ to $1 \times 10^{6} L_{\odot}$, with a peak at about $3 \times 10^{5} L_{\odot}$. The distribution is in agreement with the IRAS luminosity distribution reported, e.g., by Wood \& Churchwell (1989) for their sample of UCHII regions, while the average value is about an order of magnitude higher with respect to the sample of pre-UC candidates investigated by Molinari et al. (1996) and Sridharan et al. (2002). This could be an evolutionary effect if the pre-UC sample is made up of protostars which derive their luminosity from accretion as suggested by Sridharan et al. (2002). Another possibility is that this difference is due to the selection criteria used by Sridharan et al. (2002): in an attempt to to select UCHII region precursor they have chosen sources associated with high-density clumps but not with $\mathrm{cm}$-emission thus perhaps biasing their sample towards later-type and hence less luminous stars. Unfortunately the present data do not allow us to discriminate between the two hypotheses. Only the maser sources seem to be slightly more luminous than the non-maser ones, the median luminosities being $3.4 \times 10^{5}$ and $1.2 \times 10^{5} L_{\odot}$ for masers and non-masers, respectively. This effect could be explained by the fact that the $\mathrm{H}_{2} \mathrm{O}$ maser detection rate depends on the luminosity and that maser and FIR luminosities are well correlated (see e.g. Felli et al. 1992; Palagi et al. 1993). Using such a correlation we have calculated the expected maser flux from the corresponding distance and FIR flux: the values are above the detection limits $(3 \sigma)$ at 6.7 and $22.2 \mathrm{GHz}$ (4 and $5 \mathrm{Jy}$, respectively) reached in the survey of Codella \& Moscadelli (2000) indicating that the lack of maser detection is not due to insufficient sensitivity. Consequently, we assume that the present analysis is not affected by a luminosity bias.

\subsection{Derived gas parameters: Optical depths and temperatures}

The comparison between ${ }^{13} \mathrm{CO}$ and $\mathrm{C}^{18} \mathrm{O}$ spectra can be used also to determine some physical parameters and thus characterise the environments of our sources. For each source and transition, an estimate of the optical depth has been obtained 

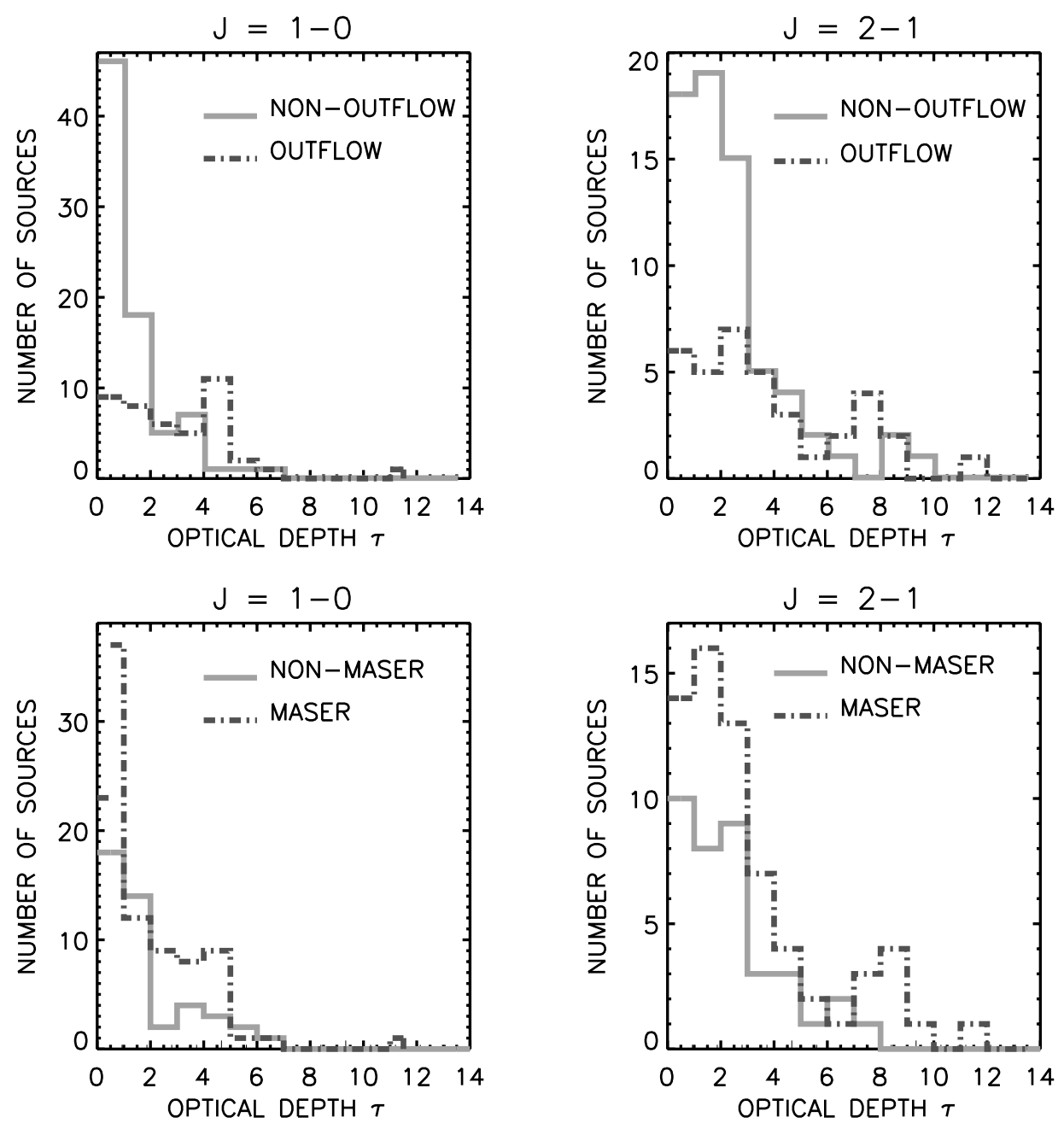

Fig. 3. Distributions of the optical depths of the ${ }^{13} \mathrm{CO} J=1-0$ (left panels) and 2-1 (right panels) lines. The two upper panels report the distribution of the outflow (dot-dashed contour) and non-outflow (continuous) subsamples. The two lower panels show the distributions of the maser (dot-dashed) and non-maser (continuous) subsamples.

from the ratio between the integrated intensities of the lines of the two isotopomers observed. Figure 3 shows the distributions of the optical depths of the $J=1-0\left(\tau_{1-0}\right.$; left panels) and $2-1\left(\tau_{2-1}\right.$; right panels) ${ }^{13} \mathrm{CO}$ lines for the outflow and non-outflow subsamples (see upper panels) and for the maser and non-maser subsamples (see lower panels). The uncertainties are $\sim 16 \%$ and $34 \%$ for $\tau_{1-0}$ and $\tau_{2-1}$, respectively. The environment of UCH II regions associated with outflows is on average more optically thick than that of sources where no outflow has been detected. On the other hand, given the large uncertainties, there is no clear indication that the same result holds also for the maser/non-maser samples. The median values of the distributions reported in Fig. 2 are: $\tau_{1-0}=2.9$ and $\tau_{2-1}=3.3$ for the outflow subsample, and $\tau_{1-0}=0.9$ and $\tau_{2-1}=1.8$ for the non-outflow subsample. For the maser and non-maser subsamples we derive respectively: $\tau_{1-0}=1.4$, $\tau_{2-1}=2.7$ and $\tau_{1-0}=1.4, \tau_{2-1}=2.2$. The corresponding average molecular hydrogen column densities, calculated assuming temperatures of $10-20 \mathrm{~K}$ and $\left[\mathrm{H}_{2}\right] /\left[{ }^{13} \mathrm{CO}\right]=77 \times$ $10^{4}$ (Wilson \& Rood 1994), are in the ranges $4 \times 10^{22}-1 \times$ $10^{23}$ and $3-5 \times 10^{22} \mathrm{~cm}^{-2}$ for the outflow and non-outflow samples, respectively. These values are lower than column densities previously derived for UCHII regions $(\leq 4 \times$ $10^{23} \mathrm{~cm}^{-2}$, Churchwell et al. 1992; $2 \times 10^{22}-7 \times 10^{24} \mathrm{~cm}^{-2}$, Hunter et al. 2000) and their precursor $\left(\sim 10^{22}-10^{23} \mathrm{~cm}^{-2}\right.$, Molinari et al. 2000). However, this discrepancy is due to the temperatures derived in the present survey (see below), which are significantly smaller than those $(\sim 100 \mathrm{~K})$ assumed by Churchwell et al. (1992). The present results confirm that the molecular outflows are associated with the earliest evolutionary phases of the high-mass star forming process, when the ionising stars are deeply embedded and their natal high-density environment is not yet significantly affected by the expansion of HII regions.

The ratio between the integrated emission due to the $J=$ 2-1 and 1-0 transition of the same molecule can be used to obtain an estimate of the rotation temperature, and hence of the kinetic temperature, assuming LTE and optically thin conditions. In this case, we choose the ${ }^{13} \mathrm{CO}$ isotopomer, which has the best $\mathrm{S} / \mathrm{N}$. One must be cautious about temperature estimates derived from ${ }^{13} \mathrm{CO}$ since the $1-0$ and $2-1$ lines have been taken with different $H P B W$ s and their ratio should be corrected for the unknown beam filling factors. Moreover, as indicated by the optical depths shown in Fig. 3, the assumption of optically 

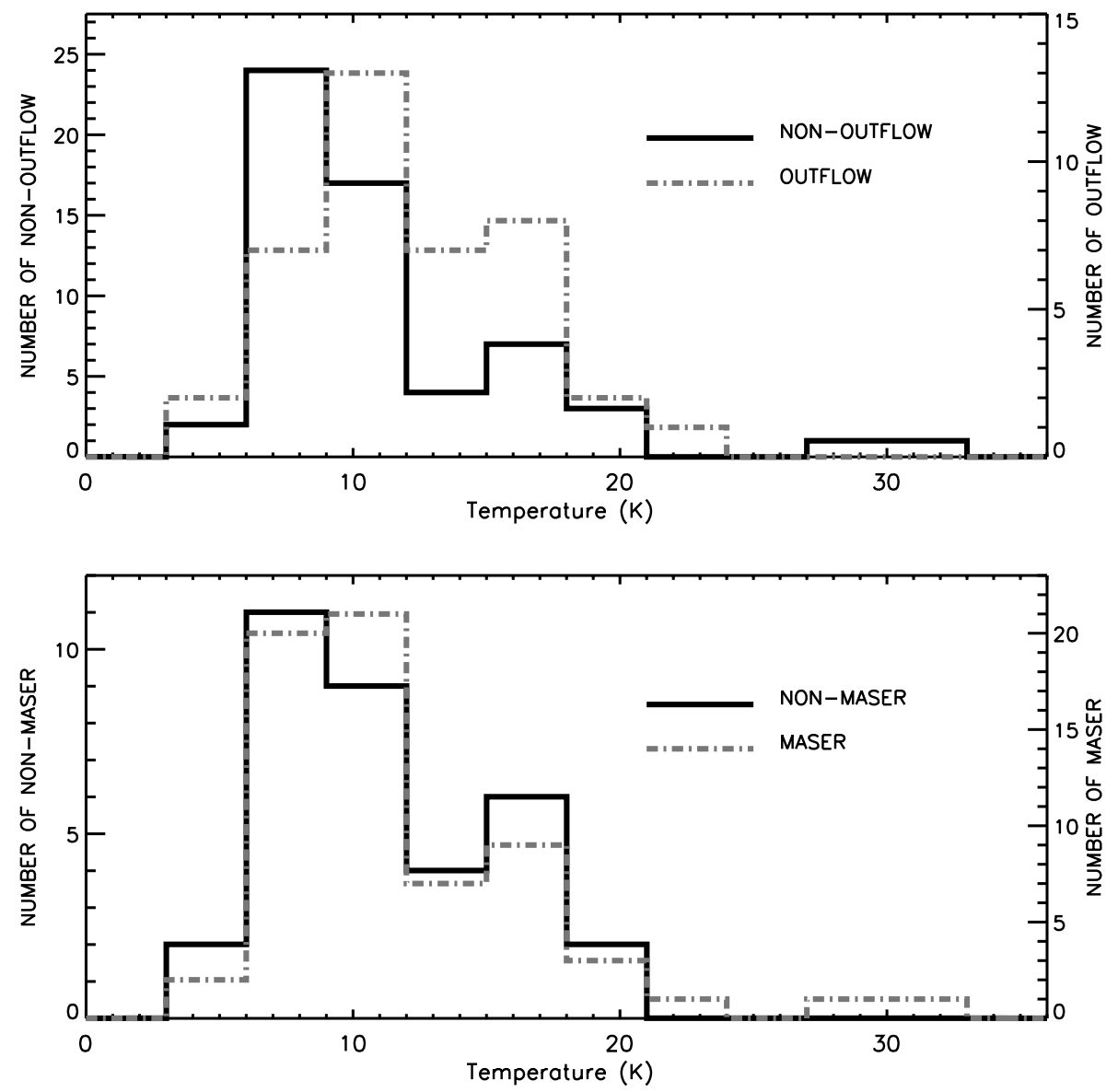

Fig. 4. Distributions of the temperature. The upper panel reports the distributions of the outflow (dot-dashed contour, left verticale scale) and non-outflow (continuous, right vertical scale) subsamples. The lower panel shows the distributions of the maser (dot-dashed, left verticale scale) and non-maser (continuous, right vertical scale) subsamples.

thin conditions for most sources is unrealistic. Therefore, when the lines turn out to be optically thin the ratio between the line areas has been used, whereas in case of optically thick lines the ratio between the corresponding optical depths has been taken. The uncertainties are $\sim 30 \%$. Figure 4 shows the result for the four subsamples: the majority of the sources have temperatures $\leq 20 \mathrm{~K}$. Of course, these values are not representative of the material close to the newly borned stars. Given the molecular tracers used here, it is plausible that we are sampling more external and colder parts of the environment hosting the star formation process. The temperatures obtained have been compared to those derived by Churchwell et al. (1990) from $\mathrm{NH}_{3}$ observations for a sample of UCHII regions. Although the overlap between the two samples regards very few cases, the temperatures from ammonia are on average larger than those from ${ }^{13} \mathrm{CO}$ by a factor of $\sim 2.5$, consistent with the common belief that $\mathrm{NH}_{3}$ traces a warmer part of the molecular cloud, closer to the newly born stars.

\subsection{An evolutionary sequence for $\mathrm{H}_{2} \mathrm{O}$ and $\mathrm{CH}_{3} \mathrm{OH}$ masers?}

The observations presented in the previous sections allow us to improve our knowledge about the association between the masers and the early evolutionary phases of the formation of massive stars associated with UCHII regions. In an attempt to interpret the results we introduce four classes of high-mass star forming regions which satisfy the following observational criteria: (1) association with masers and no association with outflows, (2) association with masers and outflows, (3) no association with masers and association with outflows, and (4) no association with masers and outflows.

Do these four classes outline an evolutionary sequence? As already pointed out in Sect. 1, we have selected massive YSOs evolved enough to create UCHII regions. In other words, the four classes defined above must be considered in a wider framework of the evolution of high-mass stars, of which they represent only a part. The pre-UC phases have been studied by other means, e.g. masers, dust IR cocoons, and hot cores, which trace earlier phases than UCHII regions. If we assume that no luminosity bias has been introduced (see Sect. 4.2) and take into account the results given in the literature, which indicate that the mass loss process is closely linked to the YSO main accretion phase (e.g. Bachiller 1996) and that the masers are associated with the earliest phases of star formation (see Sect. 1), it is tempting to interpret class 4 as the latest stage. Moreover, since the association of masers with mm-sources (Beuther et al. 2002b) tells us that masers switch on before the development of an UCHII region, we can reasonably assume classes 1 and 2 
UCHII REGION EVOLUTION

\begin{tabular}{|c|c|c|c|}
\hline (1) & (2) & (3) & (4) \\
\hline MASER: YES & MASER: YES & MASER: NO & MASER: NO \\
\hline OUTFLOW: NOT & OUTFLOW: YES & OUTFLOW: YES & OUTFLOW: NO \\
\hline
\end{tabular}

\section{TIME}

Fig. 5. Proposed evolutionary sequence for the UCHII regions. Four subclasses are identified depending on the association with $\mathrm{H}_{2} \mathrm{O}$ and $\mathrm{CH}_{3} \mathrm{OH}$ masers and/or molecular outflows (see text).

to be the earliest. Class 1 should precede class 2 to have a continuity to the phase when the outflow is detectable with singledish observations. Therefore, as summarised in Fig. 5, we tentatively propose that classes $1,2,3$, and 4 reflect four evolutionary phases sorted in order of increasing age. Thus, the results of the present survey suggest to anticipate the end of the maser phase with respect to what thought up to date: masers could disappear during the UC phase when outflows or their remnants are still present. Of course, the fact that no outflow has been detected towards the objects of class 1 does not mean a real lack of outflow motions, but instead reflect the youthness of the source: the outflow structure is too small to be detected with the angular resolution of the IRAM 30-m antenna.

Can we try to quantify the age of the class 3 objects to infer when the maser phase disappears? A rough estimate can be given by the outflow ages obtained in various surveys of massive protostars and/or UCHII regions (Shepherd \& Churchwell 1996a, 1996b; Beuther et al. 2002a): such ages generally lie between $10^{4}$ and $10^{5} \mathrm{yr}$. If we assume that these estimates are representative of the observed sample, and if we take into account that the expected life time of UCHII regions is $\sim 10^{5} \mathrm{yr}$ (Wood \& Churchwell 1989), then the maser phase should end after a few $10^{4} \mathrm{yr}$. The complexity of high-mass star forming regions and the fact that massive YSOs form in clusters strongly prevents a precise age estimate. Only detailed high angular resolution observations of a statistically significant sample of the molecular environments associated with masers and outflows will hopefully clarify this aspect. The present sample might hence represent an excellent target for forthcoming interferometric observations.

\section{Conclusions}

We have performed a search for molecular outflows towards a large homogeneous sample of UCHII regions, for which observations in the methanol $6.7 \mathrm{GHz}$ and water $22.2 \mathrm{GHz}$ maser lines had previously been obtained. The $J=1-0$ and $2-1$ transitions of ${ }^{13} \mathrm{CO}$ and $\mathrm{C}^{18} \mathrm{O}$ have been observed: the difference between the ${ }^{13} \mathrm{CO}$ and $\mathrm{C}^{18} \mathrm{O}$ line profiles of the same transitions has been used to detect prominent wings in the ${ }^{13} \mathrm{CO}$ lines. The main results are the following:

1. Out of the observed $136 \mathrm{UCHII}$ regions, 53 show occurrence of a $\mathrm{CO}$ outflow for an overall detection rate of about $39 \%$. The probability to have an outflow increases to $\sim 50 \%$ in regions where maser emission $\left(\mathrm{CH}_{3} \mathrm{OH}\right.$ and/or $\mathrm{H}_{2} \mathrm{O}$ ) has been detected, whereas in those without masers it decreases to $25 \%$. If one considers the outflow sources, the chance to find a maser is very high: $74 \%$. These values show from a statistical point of view that both $\mathrm{H}_{2} \mathrm{O}$ and $\mathrm{CH}_{3} \mathrm{OH}$ masers are closely associated with the evolutionary phase when outflows are present.

2. By comparing the ${ }^{13} \mathrm{CO}$ and $\mathrm{C}^{18} \mathrm{O}$ spectra, optical depths and temperatures have been estimated. The environment of UCHII regions associated with outflows and/or masers is on average more optically thick, confirming that these phenomena are associated with the earliest evolutionary phases, when the ionising stars are still deeply embedded in their natal high-density clumps.

3. On the basis of the maser and outflow detection rates a tentative evolutionary scheme is proposed for the high-mass star formation process. The youngest phase is that associated with maser emission and molecular outflows not yet large enough to be detected with single-dish observations. Later on, maser sources tend to fade away while the outflow expands, and finally only the UCHII region whitout masers and outflows is left.

Acknowledgements. We are grateful to F. Palagi and M. Walmsley for helpful discussions. We also thank the referee H. Beuther for comments and suggestions which have significantly improved the presentation.

\section{References}

Araya, E., Hofner, P., Churchwell, E., \& Kurtz, S. 2002, ApJS, 138, 63

Bachiller, R. 1996, ARA\&A, 34, 111

Beuther, H., Schilke, P., Sridharan, T. K., et al. 2002a, A\&A, 383, 892

Beuther, H., Walsh, A., Schilke, P., et al. 2002b, A\&A, 390, 289 
Brand, J. 1986, Ph.D. Thesis, Leiden University

Caswell, J. L., Vaile, R. A., Ellingsen, S. P., Witheoak, J. B., \& Norris, R. P. 1995, MNRAS, 272, 96

Cesaroni, R. 1990, A\&A, 233, 513

Churchwell, E., Walmsley, C. M., \& Cesaroni, R. 1990, A\&AS, 83, 119

Churchwell, E., Walmsley, C. M., \& Wood, D. O. S. 1992, A\&A, 253, 541

Codella, C., \& Moscadelli, L. 2000, A\&A, 362, 723

Codella, C., Testi, L., \& Cesaroni, R. 1997, A\&A, 325, 725

Downes, D., Wilson, T. L., Bieging, J., \& Wink, J. 1980, A\&AS, 40, 379

Ellingsen, S. P., Norris, R. P., \& McCulloch, P. M. 1996, MNRAS, 279, 101

Felli, M., Palagi, F., \& Tofani, G. 1992, A\&A, 255, 293

Henning, Th., Pfau, W., \& Altenhoff, W. J. 1990, A\&A, 227, 542

Hunter, T. R., Churchwell, E., Watson, C., et al. 2000, AJ, 119, 2711

Kurtz, S., Cesaroni, R., Churchwell, E., Hofner, P., \& Walmsley, C. M. 2000, in Protostars and Planets IV, ed. V. Mannings, A. Boss, \& S. Russell (Tucson: Univ. of Arizona Press)

Minier, V., Booth, R. S., \& Conway, J. E. 2000, A\&A, 362, 1093

Molinari, S., Brand, J., Cesaroni, R., \& Palla, F. 1996, A\&A, 308, 573

Molinari, S., Brand, J., Cesaroni, R., \& Palla, F. 2000, A\&A, 355, 617

Moscadelli, L., Cesaroni, R., \& Rioja, M. 2000, A\&A, 360, 663

Norris, R. P., Byleveld, S. E., Diamond, P. J., et al. 1998, ApJ, 508, 275

Palagi, F., Cesaroni, R., Comoretto, G., Felli, M., \& Natale, V. 1993, A\&AS, 101, 153
Seth, A. C., Greenhill, L. J., \& Holder, B. P. 2002, A\&AS, 200, 8503

Shepherd, D. S., \& Churchwell, E. 1996a, ApJ, 457, 267

Shepherd, D. S., \& Churchwell, E. 1996b, ApJ, 472, 255

Slysh, V. I., Val'tts, I. E., Kalenskii, S. V., et al. 1999, A\&AS, 134, 115

Solomon, P. M., Rivolo, A. R., Barrett, J., \& Yahil, A. 1987, ApJ, 319, 730

Sridharan, T. K., Beuther, H., Schilke, P., Menten, K. M., \& Wyrowski, F. 2002, ApJ, 566, 931

Szymczak, M., Hrynek, G., \& Kus, A. J. 2000, A\&AS, 143, 269

Tofani, G., Felli, M., Taylor, G. B., \& Hunter, T. R. 1995, A\&AS, 112, 299

Torrelles, J. M., Gómez, J. F., Rodríguez, L. F., et al. 1997, ApJ, 489, 744

Torrelles, J. M., Patel, N. A., Gómez, J. F., et al. 2001, ApJ, 560, 853

Walsh, A. J., Burton, M. G., Hyland, A. R., \& Robinson, G. 1998, MNRAS, 301, 640

Walsh, A. J., Macdonald, G. H., Alvey, N. D. S, Burton, M. G., \& Lee, J.-K. 2003, A\&A, 410, 597

Watson, C., Araya, E., Sewilo, M., et al. 2003, ApJ, 587, 714

White, R. L., Becker, R. H., \& Helfand, D. J. 1991, ApJ, 371, 148

Wilson, T. L., \& Rood, R. T. 1994, ARA\&A, 32, 191

Wink, J. E., Altenhoff, W. J., \& Mezger, P. G. 1982, A\&A, 108, 227

Wood, D. O. S., \& Churchwell, E. 1989a, ApJ, 340, 265

Wood, D. O. S., \& Churchwell, E. 1989b, ApJS, 69, 831

Zhang, Q., Hunter, T. R., Brand, J., et al. 2001, ApJ, 552, L167

Zoonematkermani, S., Helfand, D. J., Becker, R. H., White, R. L., \& Perley, R. A. 1990, ApJS, 74, 181 